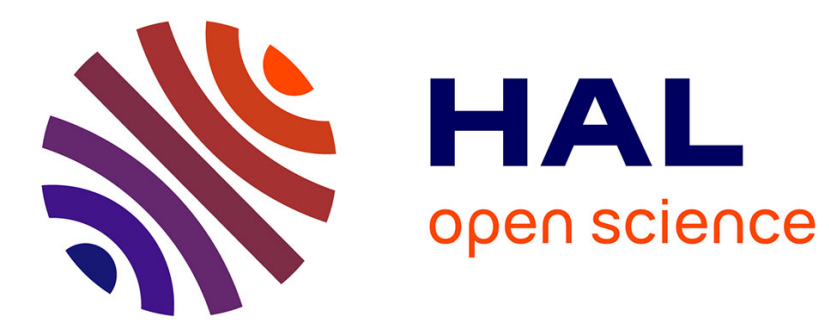

\title{
VAN DER WAALS DISPERSION FORCE CONTRIBUTION TO THE INTERFACIAL FREE ENERGY OF NEMATIC LIQUID CRYSTALS
}

K. Okano, J. Murakami

\section{- To cite this version:}

K. Okano, J. Murakami. VAN DER WAALS DISPERSION FORCE CONTRIBUTION TO THE INTERFACIAL FREE ENERGY OF NEMATIC LIQUID CRYSTALS. Journal de Physique Colloques, 1979, 40 (C3), pp.C3-525-C3-528. 10.1051/jphyscol:19793105 • jpa-00218706

\section{HAL Id: jpa-00218706 https://hal.science/jpa-00218706}

Submitted on 1 Jan 1979

HAL is a multi-disciplinary open access archive for the deposit and dissemination of scientific research documents, whether they are published or not. The documents may come from teaching and research institutions in France or abroad, or from public or private research centers.
L'archive ouverte pluridisciplinaire HAL, est destinée au dépôt et à la diffusion de documents scientifiques de niveau recherche, publiés ou non, émanant des établissements d'enseignement et de recherche français ou étrangers, des laboratoires publics ou privés. 


\title{
VAN DER WAALS DISPERSION FORCE CONTRIBUTION TO THE INTERFACIAL FREE ENERGY OF NEMATIC LIQUID CRYSTALS
}

\author{
K. OKANO and J. MURAKAMI \\ Department of Applied Physics, Faculty of Engineering, University of Tokyo, Japan
}

\begin{abstract}
Résumé. - En utilisant la théorie macroscopique des forces de van der Waals on calcule l'énergie libre superficielle à la surface libre d'un cristal liquide nématique et l'énergie libre interfacielle de nématique sur la surface de verre, pour les cas où le directeur est parallèle et perpendiculaire à la surface.
\end{abstract}

\begin{abstract}
By using the macroscopic theory of van der Waals forces, the surface free energy of a nematic liquid crystal and the interfacial free energy of a nematic on a glass substrate are calculated for the case where the director is parallel and perpendicular to the surface.
\end{abstract}

1. Introduction. - The surface free energy of a nematic liquid crystal and the interfacial free energy of nematic-glass interface depend on the mean orientation of molecules (the director). For example, the molecules of p-azoxyanisole (PAA) at a free surface are known to lie in the plane of the surface, while the director of PAA on a clean glass substrate is perpendicular to the interface.

In spite of the practical importance (e.g. in constructing display devices) of these phenomena we have, at present, no satisfactory theory to explain what physical mechanism is responsible for these phenomena $\left({ }^{1}\right)$.

In this paper we consider the contribution of van der Waals forces to the surface and interfacial free energies in relation to the molecular orientation at the interface. We take a macroscopic approach due originally to Lifshitz [2] in which materials are regarded as continuous media. Our theory is the same in spirit as the theory of Israelachvili [3] who successfully calculated the surface tension of several hydrocarbons based upon the macroscopic van der Waals dispersion forces $\left({ }^{2}\right)$.

In section 2 we calculate the surface free energies of a nematic at the free surface for the cases where the director is parallel as well as perpendicular to the surface. In section 3 we study the free energies at a nematic-glass interface for parallel and perpendicular alignments.

$\left.{ }^{1}\right)$ For a grooved surface the elastic theory is successful in explaining the parallel alignment of molecules along the groove [1], but what we are concerned here is the alignment at a completely flat surface, in which the elastic theory loses its power.

${ }^{2}$ ) See also some recent theoretical works [4], [5], [6] and [7].
2. Surface free energy. - First we discuss the case of an isotropic liquid as an illustration of our method. The starting point is the following equation for the van der Waals interaction free energy (per unit area) of semi-infinite media interacting across a plane parallel vacuum gap of width $l[8]$

$$
\begin{aligned}
U(l)= & \frac{\hbar}{4 \pi^{2}} \int_{0}^{\infty} \mathrm{d} \omega \times \\
& \times \int_{0}^{k_{\mathrm{c}}} k \mathrm{~d} k \ln \left[1-\Delta^{2}(i \omega) \exp (-2 k l)\right],
\end{aligned}
$$

where $\hbar$ is the Planck constant divided by $2 \pi$, and $k_{\mathrm{c}}$ is a cut-off wave number of fluctuating electric field along the surfaces. $\Delta(\omega)$ is related to the complex dielectric permittivity of the liquid, $\varepsilon(\omega)$, in the following way,

$$
\Delta(\omega)=\frac{\varepsilon(\omega)-1}{\varepsilon(\omega)+1} .
$$

Here the zero point of the free energy is taken as

$$
U(\infty)=0 \text {. }
$$

Now, starting from $l=\infty$ we decrease the separation $l$ and eventually we make $l=0$ in which both media are in molecular contact. Then we have the free energy $U(0)$. But this is just the reverse process of making free surfaces in the bulk medium and taking the surfaces infinitely distant apart. The work necessary for the latter process is twice the surface energy $\gamma$. So we have the fundamental relation :

$$
\gamma=-\frac{1}{2} U(0) \text {. }
$$


Combining (1) and (4), we have the equation for the surface free energy of an isotropic liquid :

$$
\gamma=-\frac{\hbar}{16 \pi^{2}} k_{\mathrm{c}}^{2} \int_{0}^{\infty} \mathrm{d} \omega \ln \left[1-\Delta^{2}(i \omega)\right]
$$

which was derived by Mitchell and Richmond [9]. Similar method was used by Israelachvili [3] for the calculation of the surface free energy of hydrocarbons.

The extension of the above method to the calculation of the surface free energy of nematic liquid crystals in the parallel and perpendicular alignments are straightforward. The only difference is that the dielectric permittivity of the interacting media is now anisotropic.

We choose the cartesian coordinate system in which $z$-axis is perpendicular to the surfaces and $x$ and $y$-axis are parallel to the surfaces. Let the dielectric permittivity tensor of the nematic be $\varepsilon(\omega)$ :

$$
\varepsilon(\omega)=\left(\begin{array}{ccc}
\varepsilon_{x}(\omega) & 0 & 0 \\
0 & \varepsilon_{v}(\omega) & 0 \\
0 & 0 & \varepsilon_{z}(\omega)
\end{array}\right)
$$

Again we begin with the van der Waals free energy of semi-infinite nematic liquid crystals interacting across a plane parallel vacuum gap of width $l$. The relevant free energy is calculated by using the method of surface mode analysis first introduced by van Kampen, Nijboer and Schram [8] and later extended by Ningam, Parsegian and Weiss [10]. Since we are interested in the non-retarded limit, the surface mode analysis reduces to an electrostatic boundary value problem.

Let the complex dielectric permittivity of the nematic in the direction parallel to the director be $\varepsilon_{\|}(\omega)$ and that perpendicular to the director be $\varepsilon_{\perp}(\omega)$, then we have for a parallel alignment

$$
\varepsilon_{x}(\omega)=\varepsilon_{\|}(\omega), \quad \varepsilon_{y}(\omega)=\varepsilon_{z}(\omega)=\varepsilon_{\perp}(\omega)
$$

whereas for a perpendicular alignment we have

$$
\varepsilon_{x}(\omega)=\varepsilon_{y}(\omega)=\varepsilon_{\perp}(\omega), \quad \varepsilon_{z}(\omega)=\varepsilon_{\|}(\omega) .
$$

And we obtain for a parallel orientation

$$
\begin{aligned}
& U_{\|}(0)=\frac{\hbar}{16 \pi^{3}} k_{\mathrm{c}}^{2} \int_{0}^{\infty} \mathrm{d} \omega \int_{0}^{2 \pi} \mathrm{d} \varphi \times \\
& \times \ln \left\{\frac{4\left[\varepsilon_{\|}(i \omega) \varepsilon_{\perp}(i \omega) \cos ^{2} \varphi+\varepsilon_{\perp}(i \omega)^{2} \sin ^{2} \varphi\right]^{1 / 2}}{\left[\left(\varepsilon_{\|}(i \omega) \varepsilon_{\perp}(i \omega) \cos ^{2} \varphi+\varepsilon_{\perp}(i \omega)^{2} \sin ^{2} \varphi\right)^{1 / 2}+1\right]}\right.
\end{aligned}
$$

and

$$
U_{\perp}(0)=\frac{\hbar}{8 \pi^{2}} k_{c}^{2} \int_{0}^{\infty} \mathrm{d} \omega \ln \left\{\frac{4\left[\varepsilon_{\|}(i \omega) \varepsilon_{\perp}(i \omega)\right]^{1 / 2}}{\left[\left(\varepsilon_{\|}(i \omega) \varepsilon_{\perp}(i \omega)\right)^{1 / 2}+1\right]^{2}}\right\}
$$

for a perpendicular orientation. Therefore the surface free energies of parallel $\left(\gamma_{\|}\right)$and perpendicular $\left(\gamma_{1}\right)$ alignments are obtained respectively as

and

$$
\gamma_{\|}=-\frac{1}{2} U_{\|}(0)
$$

$$
\gamma_{\perp}=-\frac{1}{2} U_{\perp}(0) \text {. }
$$

Fortunately in the case of PAA at $125^{\circ} \mathrm{C}$ we have the pertinent complex permittivity tensor from the radio frequency to the ultraviolet (UV) region. We employed the numerical values shown in table $1 a$ which was cited from the reference [4], and performed the numerical calculation of (9) and (10). And we obtain $U_{\|}(0)=-75.66 \mathrm{erg} / \mathrm{cm}^{2}$, and

$$
U_{\perp}(0)=-89.26 \mathrm{erg} / \mathrm{cm}^{2},
$$

where we have put $\lambda_{\mathrm{c}}=2 \pi / k_{\mathrm{c}}=10 \AA$. So that we have $\gamma_{\|}=37.8 \mathrm{erg} / \mathrm{cm}^{2}$ and $\gamma_{\perp}=44.6 \mathrm{erg} / \mathrm{cm}^{2}$. We see that $\gamma_{\|}<\gamma_{\perp}$, and the anchoring energy is $\gamma_{\|}-\gamma_{\perp}=6.8 \mathrm{erg} / \mathrm{cm}^{2}$. These theoretical results are consistent with experimental facts that the molecules of PAA lie in the plane of free surface and the values of surface free energy is $\gamma_{\text {exp }}=38 \sim 39 \mathrm{erg} / \mathrm{cm}^{2}$ at $125^{\circ} \mathrm{C}[11],[12]$.

3. Free energy of nematic-glass interface. - Next let us discuss the case of nematic on a clean, flat glass substrate. Again we begin with the van der Waals free energy between the semi-infinite media interacting across a plane parallel vacuum gap of width $l$. Here one of the media is a nematic and the other is a glass.

The surface mode analysis gives the following equations of interaction free energy at $l=0$ for parallel and perpendicular alignments respectively

$U_{\|}(0)=\frac{\hbar}{16 \pi^{3}} k_{\mathrm{c}}^{2} \int_{0}^{\infty} \mathrm{d} \omega \int_{0}^{2 \pi} \mathrm{d} \varphi \ln \left[1-\Delta_{\|}^{2}(i \omega)\right]$,

where

$$
\begin{aligned}
& \Delta_{\|}^{2}(\omega)=\left[\frac{\varepsilon_{\mathrm{g}}(\omega)-1}{\varepsilon_{\mathrm{g}}(\omega)+1}\right] \times \\
& \quad \times\left\{\frac{\left[\varepsilon_{\|}(\omega) \varepsilon_{\perp}(\omega) \cos ^{2} \varphi+\varepsilon_{\perp}(\omega)^{2} \sin ^{2} \varphi\right]^{1 / 2}-1}{\left[\varepsilon_{\|}(\omega) \varepsilon_{\perp}(\omega) \cos ^{2} \varphi+\varepsilon_{\perp}(\omega)^{2} \sin ^{2} \varphi\right]^{1 / 2}+1}\right\},
\end{aligned}
$$

and

$$
U_{\perp}(0)=\frac{\hbar}{8 \pi^{2}} k_{\mathrm{c}}^{2} \int_{0}^{\infty} \mathrm{d} \omega \ln \left[1-\Delta_{\perp}^{2}(i \omega)\right],
$$

where

$$
\Delta_{\perp}^{2}(\omega)=\left[\frac{\varepsilon_{\mathrm{g}}(\omega)-1}{\varepsilon_{\mathrm{g}}(\omega)+1}\right]\left\{\frac{\left[\varepsilon_{\|}(\omega) \varepsilon_{\perp}(\omega)\right]^{1 / 2}-1}{\left[\varepsilon_{\|}(\omega) \varepsilon_{\perp}(\omega)\right]^{1 / 2}+1}\right\}
$$

In the above equations $\varepsilon_{\mathrm{g}}(\omega)$ is the complex dielectric permittivity of the glass substrate.

If we denote the interfacial free energy for a parallel orientation by $\gamma_{\|}^{\prime}$ and that for the perpendicular 
orientation by $\gamma_{\perp}^{\prime}$, and if the surface free energy (the free energy at the free surface) of the glass substrate be $\gamma_{\mathrm{g}}$, then we have the following relations

and

$$
\gamma_{\|}^{\prime}=\gamma_{\|}+\gamma_{\mathrm{g}}+U_{\|}(0),
$$

$$
\gamma_{\perp}^{\prime}=\gamma_{\perp}+\gamma_{\mathbf{g}}+U_{\perp}(0)
$$

Here $U_{\|}(0)$ and $U_{\perp}(0)$ are respectively given by (13) and (15), and $\gamma_{\|}$and $\gamma_{\perp}$ correspond respectively to (11) and (12).

So that we have the expression of the anchoring energy at the nematic-glass interface :

$$
\gamma_{\|}^{\prime}-\gamma_{\perp}^{\prime}=\gamma_{\|}-\gamma_{\perp}+U_{\|}(0)-U_{\perp}(0) .
$$

We also performed the numerical calculation of the above $U_{\|}(0)$ and $U_{\perp}(0)$ for PAA (at $125^{\circ} \mathrm{C}$ ) and found $U_{\|}(0)=-77.89 \mathrm{erg} / \mathrm{cm}^{2}$ and

$$
U_{\perp}(0)=-85.05 \mathrm{erg} / \mathrm{cm}^{2} \text {. }
$$

Here again we have employed the complex permittivities of PAA (Table $\mathrm{I} a$ ) and the glass (Table $\mathbf{1} b$ ) as those used in the reference [4]. (The permittivity of the model glass $\mathrm{A}$ of the paper cited was used.) In the above calculation we also put

$$
\lambda_{\mathrm{c}}=2 \pi / k_{\mathrm{c}}=10 \mathrm{~A} .
$$

And we found the theoretical anchoring energy : $\gamma_{\|}^{\prime}-\gamma_{\perp}^{\prime}=0.36 \mathrm{erg} / \mathrm{cm}^{2}$. This shows that on a clean flat glass surface, the molecules of PAA orient them-

\section{TABLE I}

\section{Dielectric data for PAA and a model glass}

$$
\varepsilon(\omega)=1+\sum_{\mathrm{m}} \frac{B_{\mathrm{m}}}{1-i \omega / \omega_{\mathrm{m}}}+\sum_{\mathrm{e}} \frac{C_{\mathrm{e}}}{1-\left(\omega / \omega_{\mathrm{e}}\right)^{2}}
$$

a) Dielectric data for PAA at $125^{\circ} \mathrm{C}$.

$\begin{array}{cccc}\begin{array}{c}\text { Relaxation } \\ \text { frequency } \\ \text { (rad/s) }\end{array} & B_{\mathrm{m}}, C_{\mathrm{e}} & \begin{array}{c}\text { Relaxation } \\ \text { frequency } \\ \text { (rad/s) }\end{array} & B_{\mathrm{m}}, C_{\mathrm{e}} \\ - & - & - & - \\ 3.78 \times 10^{8} & B_{1}=0.93 & 5.65 \times 10^{10} & B_{1}=3.08 \\ 5.65 \times 10^{10} & B_{2}=1.23 & 1.88 \times 10^{14} & C_{1}=0.38 \\ 1.88 \times 10^{14} & C_{1}=0.16 & 6.28 \times 10^{15} & C_{2}=1.40 \\ 6.28 \times 10^{15} & C_{2}=2.31 & & \end{array}$

b) Dielectric data for a model glass.

Relaxation

$$
\begin{array}{cc}
\begin{array}{c}
\text { frequency } \\
(\mathrm{rad} / \mathrm{s})
\end{array} & C_{\mathrm{e}} \\
- & - \\
1.0 \times 10^{14} & C_{1}=1.56 \\
1.0 \times 10^{16} & C_{2}=1.25
\end{array}
$$

selves perpendicularly to the interface which is in agreement with experimental observation [13]. However, in view of the fact that the accuracy of our calculations are not high enough as will be discussed in the next section, in particular the used dielectric data of the glass is only of a crude model, we cannot give much credit to the above figures. Nevertheless we may safely conclude that anchoring energy at the nematic-glass interface is very small as compared with that of free surface.

4. Discussion and conclusion. - Our calculations in the preceding sections are not complete in the following respects.

First, the macroscopic (Lifshitz) theory of van der Waals forces, which is only applicable for separations 1 greater than interatomic distances, was used for bodies in molecular contact. This is reflected in the fact that the resulting equations contain a cut-off wave number $k_{\mathrm{c}}$ as an adjustable parameter. The Lifshitz theory, however, has been found to yield rather good results in molecular liquids even when used at separations of the order of interatomic spacings [3]. In addition, our choice of

$$
\lambda_{\mathrm{c}}=2 \pi / k_{\mathrm{c}}=10 \mathrm{~A}
$$

is not unreasonable, since the value roughly corresponds to the mean size of a PAA molecule.

Second, we have only considered the cases of parallel and perpendicular alignments. An extension to the case of oblique alignment is not impossible in principle, but the calculation becomes very complicated.

Third, the data of complex dielectric permittivity tensor of PAA (and also of glass substrate) are not complete. There must be some absorption spectra beyond the UV region. This can be infered from the fact that the used data up to the UV region does not lead to the plasma frequency [4], which determines the refractive index in the $\mathrm{X}$-ray region. Although the contribution of this absorption beyond the UV region to the van der Waals interaction is expected not so large, it may affect the result of anchoring energy of nematic on a glass substrate to some extent where, as we have seen in the previous section, the anchoring energy itself is very small.

In spite of these defects, the present theory may be of use for understanding the physical mechanism of interfacial phenomena in liquid crystals. We may conclude that the surface free energy of a nematic and the interfacial free energy of a nematic on a flat and clean substrate (the anchoring energy in particular) are considered to be closely related to the complex dielectric permittivity tensors of the nematic and the substrate at UV region and even at higher frequencies. 


\section{References}

[1] Berreman, D., Phys. Rev. Lett. 28 (1972) 1683 ; See also : De Gennes, P. G., The Physics of Liquid Crystals, Oxford University Press, 1974.

[2] LifShiTz, E. M., Sov. Phys. JETP 2 (1958) 73.

[3] Israelachyil, J. N., J. Chem. Soc., Faraday Trans. II 69 (1973) 1729.

[4] Richmond, P. and White, L. R., Mol. Cryst. Liq. Cryst. 27 (1974) 217.

[5] Dubois-Violette, E. and de Gennes, P. G., J. Physique Lett. 36 (1975) L-255.

[6] Dubois-Violette, E. and de Gennes, P. G., J. Colloid Interface Sci. 57 (1976) 403.
[7] Parsons, J. D., J. Physique 37 (1976) 1187.

[8] Van Kampen, N. G., Nijboer, B. R. A. and Schram, K., Phys. Lett. A 26 (1968) 307.

[9] Mitchell, D. J. and Richmond, P., Chem. Phys. Lett. 21 (1973) 113.

[10] Ninham, B. W., Parsegian, V. A. and Weiss, G. H., J. Stat. Phys. 2 (1970) 323.

[11] Ferguson, A. and Kennedy, S. J., Phil. Mag. 26 (1938) 41.

[12] Schwartz, M. W. and Moselex, H. W., J. Colloid. Chem. 51 (1947) 826.

[13] See e.g. Goodman, L. A., RCA Rev. 35 (1974) 447. 